\title{
AMENABILITY OF WEIGHTED CONVOLUTION ALGEBRAS ON LOCALLY COMPACT GROUPS
}

\author{
NIELS GRØNBÆK
}

\begin{abstract}
We give a direct transition from the existence of a bounded right approximate identity in the diagonal ideal for a weighted convolution algebra on a locally compact group to the existence of translation invariant means on an associated weighted $L^{\infty}$-space, thus giving a characterization of amenability for such an algebra.
\end{abstract}

\section{INTRODUCTION}

A Banach algebra $\mathfrak{A}$ is called amenable if $H^{1}\left(\mathfrak{A}, X^{*}\right)=0$ for all Banach $\mathfrak{A}$-bimodules $X$. This concept was introduced by B. E. Johnson in [5]. In this paper Johnson showed that $L^{1}(G)$ is amenable if and only if $G$ is amenable, i.e. if and only if there is a (left-)translation invariant mean on $L^{\infty}(G)$. He also showed that amenable Banach algebras have bounded approximate identities and that $L^{1}(G)$ is amenable if and only if the augmentation ideal $I_{0}=\{f \in$ $\left.L^{1}(G) \mid \int_{G} f=0\right\}$ has a bounded right approximate identity. In the sequel we shall use the abbreviation 'BAI' for 'bounded two-sided approximate identity', and 'BRAI' and 'BLAI' for the one-sided concepts.

The importance of approximate identities for amenability was clarified by A. Ya. Khelemskii and M. V. Sheinnberg (see the survey article [6]). In a series of papers they developed a general homology theory for Banach algebras and obtained the following description of amenability. Given a Banach algebra $\mathfrak{A}$, they considered the sequence

$$
0 \rightarrow \operatorname{ker} \pi \stackrel{i}{\rightarrow} \mathfrak{A} \widehat{\otimes} \mathfrak{A}^{o p} \stackrel{\pi}{\rightarrow} \mathfrak{A} \rightarrow 0,
$$

where $\widehat{\otimes}$ is the projective tensor product, the Banach algebra $\mathfrak{A}^{o p}$ is obtained by reversing the product in $\mathfrak{A}$, the map $\pi$ is given by $\pi(a \otimes b)=a b$, and the map $i$ is the injection of the kernel of $\pi$. With the usual multiplication on $\mathfrak{A} \widehat{\otimes} \mathfrak{A}^{o p}$, the set ker $\pi$ becomes a closed left-ideal of $\mathfrak{A} \widehat{\otimes} \mathfrak{A}^{o p}$, called the diagonal ideal.

Received by the editors February 22, 1988 and, in revised form, September 7, 1988.

1980 Mathematics Subject Classification (1985 Revision). Primary 46H20; Secondary 43A20, $43 \mathrm{~A} 07$.

Key words and phrases. Amenable, bounded approximate identity, convolution, diagonal ideal, translation invariant mean. 
Theorem (Khelemskii and Sheinberg). $\mathfrak{A}$ is amenable if and only if $\mathfrak{A}$ has a BAI and the diagonal ideal has a BRAI.

This theorem has recently been reproved with only a minimum of homological methods by P. C. Curtis, Jr. and R. J. Loy [2] and it is of some interest directly to establish the connection from a BRAI in the diagonal ideal to other (intrinsic) criteriae for amenability. This was done in [4] for a class of weighted discrete semigroup algebras.

In this paper we study weighted convolution algebras on locally compact groups. For a fixed group, the various weighted group algebras are isomorphic as Banach spaces, but quite different as Banach algebras. We relate the existence of a BRAI in the diagonal ideal to amenability of the group and growth conditions on the weight. In the discrete case [4] this could be done by essentially combinatorial methods, but in the nondiscrete case we have to rely more deeply on properties of integration with respect to Haar measures. These properties and other technical results are dealt with in $\S 1$. In $\S 2$ we prove the main theorem, which reads

Theorem $\mathbf{0}$. The following statements are equivalent:

(a) $L^{1}(\omega)$ is amenable.

(b) $L^{1}(\Omega)$ is amenable.

(c) There is a bounded net $\left(\mu_{i}\right)_{i \in I}$ in $L^{1}(\Omega)$ such that $\int_{G} \mu_{i}(s) d s \rightarrow 1$ and $\left\|\delta_{r} \cdot \mu_{i}-\mu_{i}\right\| \rightarrow 0 \quad(r \in G)$.

(d) There is a left-invariant mean on $L^{\infty}\left(\Omega^{-1}\right)$.

(e) $G$ is amenable and $\sup \{\Omega(g) \mid g \in G\}<\infty$.

Here $L^{1}(\omega)$ is a weighted group algebra, and $\Omega(g)=\omega(g) \omega\left(g^{-1}\right) \quad(g \in$ $G)$ is an auxillary weight; see the details in $\S 1$. The crux of the matter is the implication $(a) \Leftrightarrow(d)$. An invariant mean is obtained directly from a BRAI in the diagonal ideal as a weak*-limit of vector valued integrals with kernel from the BRAI. Conversely, a BRAI in the diagonal ideal is obtained from an invariant mean by constructing a right unit for the double conjugate of the diagonal ideal. These proofs are fairly easy in the unweighted case, but certain estimates are complicated by the fact that the weight may be strictly submultiplicative.

\section{Preliminaries}

In this section we make the basic definitions and give some elementary properties of convolution in weighted spaces. Most of the statements of this section are well known in the unweighted case. However, the weighted cases do not follow trivially from the corresponding statements in the unweighted case, so we give proofs in some detail.

Definition 1.1. Let $G$ be a locally compact group and let $\rho: G \rightarrow] 0, \infty[$ be a continuous function. Let $X$ be a Banach space of measures or (equivalence classes of) functions on $G$. We define the corresponding weighted space as 
$X(\rho)=\{f \mid \rho f \in X\}$. We norm $X(\rho)$ so that the map $f \mapsto \rho f: X(\rho) \rightarrow X$ becomes an isometry.

The spaces $X$ that we shall use are:

$$
\begin{aligned}
M(G) & =\{\mu \mid \mu \text { is a regular Borel measure, }\|\mu\|=|\mu|(G)<\infty\}, \\
L^{1}(G) & =\{\mu \mid \mu \text { is absolutely continuous with } \\
\quad \text { respect to left-invariant Haar measure }\}, & \\
L^{\infty}(G) & =\{f \mid f \text { Borel measurable, }\|f\|=\text { ess sup }\{|f(t)| \mid t \in G\}\}, \\
C_{\mathrm{ruc}}(G) & =\left\{f \in L^{\infty}(G) \mid f \text { is right uniformly continuous }\right\}, \\
C_{\text {luc }}(G) & =\left\{f \in L^{\infty}(G) \mid f \text { is left uniformly continuous }\right\}, \\
C_{\mathrm{uc}}(G) & =C_{\mathrm{ruc}}(G) \cap C_{\mathrm{luc}}(G), \\
C_{0}(G) & =\left\{f \in L^{\infty}(G) \mid f \text { is continuous, } f(t) \rightarrow 0 \text { as } t \rightarrow \infty\right\} .
\end{aligned}
$$

We shall usually suppress mentioning which group we are dealing with, when we use weighted spaces. Thus, we shall for instance write $L^{1}(\rho)$ instead of $L^{1}(G)(\rho)$. This should cause no ambiguity, since the group $G$ is implicitly given as the domain of $\rho$.

The letter $\omega$ will be used to designate a submultiplicative continuous weight on $G$, that is a continuous function $\omega: G \rightarrow] 0, \infty[$ satisfying

$$
\omega(s t) \leq \omega(s) \omega(t) \quad(s, t \in G) .
$$

For convenience we shall assume $\omega(e)=1$, where $e$ is the neutral element of $G$. With convolution of measures as algebra product $M(\omega)$ becomes a Banach algebra, having $L^{1}(\omega)$ as a closed two-sided ideal.

As described in the introduction, we will be dealing with the sequence

$$
0 \rightarrow \nabla \stackrel{i}{\rightarrow} L^{1}(\omega) \widehat{\otimes} L^{1}(\omega)^{o p} \stackrel{\pi}{\rightarrow} L^{1}(\omega) \rightarrow 0,
$$

where $\nabla$ is the diagonal ideal. Since $L^{1}(\omega)$ has a BAI, the product map $\pi$ is surjective, so that the sequence is short exact.

Let $d t$ denote left invariant Haar measure on $G$, and let $\delta_{g}$ be the Dirac measure at $g(g \in G)$. The modular function for $G$ is denoted $\Delta$. We make the cartesian product $G \times G$ a locally compact group by defining the product

$$
(s, t)(g, h)=(s g, h t) \quad(s, t, g, h \in G) .
$$

We shall always assume that $G \times G$ is equipped with this multiplication.

Proposition 1.2. $L^{1}(\omega) \hat{\otimes} L^{1}(\omega)^{o p}$ is isometrically isomorphic to $L^{1}(\omega \times \omega)$ as Banach algebras where $\omega \times \omega: G \times G \rightarrow \mathbf{R}_{+}$is given by $\omega \times \omega(s, t)=\omega(s) \omega(t)$. For $x, y \in L^{1}(\omega \times \omega)$ the product is given by

$$
\left.(x * y)(g, h)=\iint_{G \times G} x\left(g s, t^{-1} h\right) y\left(s^{-1}, t\right) d s d t \quad \text { (a.e. }(g, h)\right)
$$


and for $(g, h) \in G \times G$

$$
\begin{aligned}
& \left(\delta_{(g, h)} * x\right)(s, t)=x\left(g^{-1} s, t h^{-1}\right) \Delta\left(h^{-1}\right) \quad(\text { a.e. }(s, t)), \\
& \left(x * \delta_{(g, h)}\right)(s, t)=x\left(s g^{-1}, h^{-1} t\right) \Delta\left(g^{-1}\right) \quad(\text { a.e. }(s, t)) .
\end{aligned}
$$

Proof. The Banach space isomorphism between $L^{1}(\omega) \widehat{\otimes} L^{1}(\omega)$ and $L^{1}(\omega \times \omega)$ is well known and formulas (2), (3), and (4) are obvious for elementary tensors. By continuity of the algebra product they follow in general. The Banach algebra isomorphism is obtained by the substitution $t:=t^{-1}$ in (2) and by noting that $\Delta\left(t^{-1}\right) d s \times d t$ is a left-invariant Haar measure on $G \times G$ with the multiplication (1).

The module multiplications used in this paper are (restrictions of) the module operations obtained by dualization of the algebra product of $M(\omega)$ on $L^{1}(\omega)$ (see p. 50 of [1]).

Proposition 1.3. $L^{1}(\omega) \cdot L^{\infty}\left(\omega^{-1}\right)=C_{\mathrm{luc}}\left(\omega^{-1}\right)=\left\{\varphi \in L^{\infty}\left(\omega^{-1}\right) \mid t \rightarrow \delta_{t} \cdot \varphi\right.$ is norm-continuous at $e\}$.

Proof. Let $a \in L^{1}(\omega)$ and $f \in L^{\infty}\left(\omega^{-1}\right)$. Then for $s, t \in G$

$$
\begin{aligned}
& \left|\frac{1}{\omega(s t)} a \cdot f(s t)-\frac{1}{\omega(s)} a \cdot f(s)\right| \\
& \quad=\left|\frac{1}{\omega(s t)}\left\langle\delta_{s t} * a, f\right\rangle-\frac{1}{\omega(s)}\left\langle\delta_{s} * a, f\right\rangle\right| \\
& \quad \leq\left|\frac{1}{\omega(s t)}-\frac{1}{\omega(s)}\right|\left|\left\langle\delta_{s t} * a, f\right\rangle\right|+\frac{1}{\omega(s)}\left|\left\langle\left(\delta_{s t}-\delta_{s}\right) * a, f\right\rangle\right| \\
& \quad \leq\left|1-\frac{\omega(s t)}{\omega(s)}\right||\langle a, f\rangle|+\left\|\delta_{t} * a-a\right\|\|f\| \\
& \quad \leq\left(\max \left\{|1-\omega(t)|,\left|1-\omega\left(t^{-1}\right)^{-1}\right|\right\}\|a\|+\left\|\delta_{t} * a-a\right\|\right)\|f\|,
\end{aligned}
$$

which is a uniform bound, tending to zero as $t \rightarrow e$. Conversely, suppose that $\varphi \in C_{\text {luc }}\left(\omega^{-1}\right)$. Then we want to show that $e_{i} \cdot \varphi \rightarrow \varphi$, where $\left(e_{i}\right)_{i \in I}$ is a usual $\delta$-shaped BAI for $L^{1}(\omega)$. Cohen's factorization theorem then gives the remaining inclusion of the first equality in the proposition. Estimating in the same way as in the unweighted case gives

$$
\begin{aligned}
\frac{1}{\omega(s)}\left|\left(e_{i} \cdot \varphi\right)(s)-\varphi(s)\right| \leq & \sup \left\{|1-\omega(t)|,\left|1-\omega\left(t^{-1}\right)^{-1}\right| \mid t \in \operatorname{supp} e_{i}\right\}\|\varphi\| \\
& +\sup \left\{\left|\frac{\varphi(s t)}{\omega(s t)}-\frac{\varphi(s)}{\omega(s)}\right| t \in \operatorname{supp} e_{i}\right\} .
\end{aligned}
$$

Since $\varphi \in C_{\text {luc }}\left(\omega^{-1}\right)$, this is a uniform bound which can be made arbitrarily small by choosing supp $e_{i}$ sufficiently small. Suppose now that $t \rightarrow \delta_{t} \cdot \varphi$ is 
norm-continuous at $e$. Then for $s, t \in G$

$$
\begin{aligned}
\left|\frac{\varphi(s t)}{\omega(s t)}-\frac{\varphi(s)}{\omega(s)}\right| & \leq\left|\frac{\varphi(s t)}{\omega(s t)}-\frac{\varphi(s t)}{\omega(s)}\right|+\left|\frac{\varphi(s t)-\varphi(s)}{\omega(s)}\right| \\
& \leq\left|\frac{\varphi(s t)}{\omega(s t)}\right|\left|1-\frac{\omega(s t)}{\omega(s)}\right|+\left\|\delta_{t} \cdot \varphi-\varphi\right\| \\
& \leq\|\varphi\| \max \left\{|1-\omega(t)|,\left|1-\omega\left(t^{-1}\right)^{-1}\right|\right\}+\left\|\delta_{t} \cdot \varphi-\varphi\right\|
\end{aligned}
$$

from which it follows that $\varphi \in C_{\text {luc }}\left(\omega^{-1}\right)$. The remaining inclusion is trivial.

In the proof of the main theorem we shall make use of certain molecular measures in $M(\omega \times \omega)$, defined as

$$
Y^{s, t}=\delta_{(s, t)}-\delta_{(e, s t)} \quad(s, t \in G) .
$$

Proposition 1.4. The subspace

$$
\nabla_{0}=\operatorname{span}\left\{x * Y^{g, h} \mid g, h \in G, x \in L^{1}(\omega \times \omega)\right\}
$$

of $L^{1}(\omega \times \omega)$ is dense in the diagonal ideal.

Proof. Let $\varphi \in L^{\infty}\left((\omega \times \omega)^{-1}\right)=L^{1}(\omega \times \omega)^{*}$ and suppose $\varphi \perp \nabla_{0}$. We must prove that $\varphi \perp \nabla$. Since $L^{1}(\omega \times \omega)$ has a BAI it is enough to prove that $\varphi \cdot y \perp \nabla$ for all $y \in L^{1}(\omega \times \omega)$. Clearly $\varphi \cdot y \perp \nabla_{0}$, so we may assume, using Proposition 1.3 , that $\varphi$ is continuous. Then

$$
\begin{aligned}
0 & =\iint_{G \times G}\left(x * Y^{g, h}\right)(s, t) \varphi(s, t) d s d t \\
& =\iint_{G \times G}\left[x\left(s g^{-1}, h^{-1} t\right) \Delta\left(g^{-1}\right)-x\left(s,(g h)^{-1} t\right)\right] \varphi(s, t) d s d t \\
& =\iint_{G \times G} x(s, t)[\varphi(s g, h t)-\varphi(s, g h t)] d s d t
\end{aligned}
$$

for all $x \in L^{1}(\omega \times \omega)$ and all $g, h \in G$. It follows that $\varphi(s g, h t)=\varphi(s, g h t)$ for all $g, h, s, t \in G$. In particular with $s=t=e$ we get $\varphi(g, h)=\varphi(e, g h)$. Since $\pi^{*}(f)(s, t)=f(s t)$ for all $f \in L^{\infty}\left(\omega^{-1}\right)$ it follows that $\varphi \in \operatorname{Im} \pi^{*}$, i.e. $\varphi \perp \nabla$. To finish, we prove that $\nabla_{0} \subseteq \nabla$. This is equivalent to

$$
\left\langle\pi^{*} f, x * Y^{g, h}\right\rangle=0 \quad\left(f \in L^{\infty}\left(\omega^{-1}\right) ; x \in L^{1}(\omega \times \omega) ; g, h \in G\right) .
$$

But $Y^{g, h} \cdot \pi^{*}(f)(s, t)=\left(\pi^{*} f\right)(s g, h t)-\left(\pi^{*} f\right)(s, g h t)=0$, so this is obvious.

Let $\omega$ be a continuous submultiplicative weight on $G$. We shall need the following auxillary weights:

$$
\begin{gathered}
\tilde{\omega}(t)=\omega\left(t^{-1}\right) \quad(t \in G), \\
\Omega(t)=\omega(t) \tilde{\omega}(t) \quad(t \in G) .
\end{gathered}
$$


Lemma 1.5. Let $m \in C_{\mathrm{uc}}(\tilde{\omega})$ and let $\varphi \in C_{\mathrm{uc}}\left(\Omega^{-1}\right)$. Then $\Psi \in C_{\mathrm{ruc}}\left((\omega \times \omega)^{-1}\right)$, where

$$
\Psi(s, t)=m(s t) \varphi\left(t^{-1}\right) \quad(s, t \in G) .
$$

Proof. By Proposition 1.3 we must prove that $\lim _{(g, h) \rightarrow(e, e)}\left\|\Psi \cdot \delta_{(g, h)}-\Psi\right\|=0$. Let $s, t, g, h \in G$. Then

$$
\begin{aligned}
& \frac{|\Psi(g s, t h)-\Psi(s, t)|}{\omega(s) \omega(t)}=\frac{\mid m(g s t h) \varphi\left((t h)^{-1}\right)-m(s t) \varphi\left(t^{-1}\right)}{\omega(s) \omega(t)} \\
& \quad=\frac{\left|(m(g s t h)-m(s t)) \varphi\left((t h)^{-1}\right)+m(s t)\left(\varphi\left((t h)^{-1}\right)-\varphi\left(t^{-1}\right)\right)\right|}{\omega(s) \omega(t)} \\
& \leq \frac{\Omega\left((t h)^{-1}\right)}{\omega(s) \omega(t)}|m(g s t h)-m(s t)|\|\varphi\|+\frac{|m(s t)| \tilde{\omega}(t)}{\omega(s)}\left\|\varphi \cdot \delta_{h^{-1}}-\varphi\right\| \\
& \leq \Omega(h)\left\|\delta_{h} \cdot m \cdot \delta_{g}-m\right\|+\|m\|\left\|\varphi \cdot \delta_{h^{-1}}-\varphi\right\| .
\end{aligned}
$$

As in Proposition 1.3 we see that, if $m \in C_{\mathrm{uc}}(\tilde{\omega})$, then $(g, h) \rightarrow \delta_{h} \cdot m \cdot \delta_{g}$ is norm continuous at $(e, e)$.

In the argument giving the equivalence of left-invariant means and BRAI's it is useful to evaluate $L^{\infty}$-functions at points.

The following proposition serves as a means for that. But first we define left-invariant means. We use ' $\chi_{A}$ ' as the symbol for the characteristic function of the set $A$.

Definition 1.6. Let $\omega: G \rightarrow \mathbf{R}_{+}$be a submultiplicative continuous weight on $G$ with $\inf \{\omega(s) \mid s \in G\}>0$, and let $X$ be a closed subspace of $L^{\infty}\left(\omega^{-1}\right)$ such that $X \cdot \delta_{t} \subseteq X \quad(t \in G)$ and $\chi_{G} \in X$. A left-invariant mean on $X$ is a positive functional $M \in X^{*}$ such that $\left\langle\chi_{G}, M\right\rangle=1$ and $\delta_{t} \cdot M=M \quad(t \in G)$.

Proposition 1.7. Let $\Omega$ be as in (6). There is a left-invariant mean on $C_{\mathrm{uc}}\left(\Omega^{-1}\right)$ if and only if there is a left-invariant mean on $L^{\infty}\left(\Omega^{-1}\right)$.

Proof. This is proved exactly as the corresponding part of Theorem 2.2.1 of [3], if one uses the version of Lemma 2.2.2 in Appendix 3 of this reference. We sketch the proof.

Since

$$
\max \left\{\left|\Omega(s t)^{-1}-\Omega(t)^{-1}\right|,\left|\Omega(t s)^{-1}-\Omega(t)^{-1}\right|\right\} \leq 1-\Omega(s)^{-1},
$$

the function $\chi_{G}$ is in $C_{\mathrm{uc}}\left(\Omega^{-1}\right)$. Let $M_{0}$ be a left-invariant mean on $C_{\mathrm{uc}}\left(\Omega^{-1}\right)$. Let $p \in L^{1}(\Omega)$ satisfy $p \geq 0, \operatorname{supp} p$ compact, and $\int_{G} p d t=1$. Then $p \cdot M_{0}=$ $M_{0}$. This follows by use of the identity $\varphi \cdot p=\int \varphi \cdot \delta_{x} p(x) d x\left(\varphi \in C_{\mathrm{uc}}\left(\Omega^{-1}\right)\right)$, where the vector valued integral is weakly convergent. Now choose a compact neighbourhood $E$ of $e \in G$ such that $E=E^{-1}$ and let $p=\frac{1}{|E|} \chi_{E}$. Define $\langle f, M\rangle=\left\langle p \cdot f \cdot p, M_{0}\right\rangle \quad\left(f \in L^{\infty}\left(\Omega^{-1}\right)\right)$. Then $M$ is a left-invariant mean on $L^{\infty}\left(\Omega^{-1}\right)$.

The following lemma is used in the construction of left-invariant means. 
Lemma 1.8. Let $\rho: G \rightarrow] 0, \infty[$ be continuous and satisfy

$$
\sup \left\{\frac{\rho(g)}{\rho\left(r g r^{-1}\right)} \mid g \in G\right\}<\infty \quad(r \in G) .
$$

Put $K=\left\{m \in C_{0}(\rho) \mid \operatorname{supp} m\right.$ is compact, $\left.m \geq 0, m(e)=1,\|m\| \leq \rho(e)\right\}$. To each $\varepsilon>0$ and each finite set $\left\{r_{1}, \ldots, r_{n}\right\} \subseteq G$ there is $m \in K$ such that

$$
\max \left\{\left\|m\left(r_{i} \cdot r_{i}^{-1}\right)-m\right\| \mid i=1, \ldots, n\right\}<\varepsilon .
$$

Proof. Suppose not. Then one may choose a finite set $\left\{r_{1}, \ldots, r_{n}\right\} \subseteq G$ and $\varepsilon>0$ so that for all $m \in K$ we have $\max \left\{\left\|m\left(r_{i} \cdot r_{i}^{-1}\right)-m\right\| \mid i=1, \ldots, n\right\} \geq \varepsilon$. Define $X=C_{0}(\rho) \times \cdots \times C_{0}(\rho)(n$ copies $)$, and put $\left(T_{i} \psi\right)(t)=\psi\left(r_{i} t r_{i}^{-1}\right)-\psi(t)$ $\left(\psi \in C_{0}(\rho), t \in G, i=1, \cdots, n\right)$. Then $\mathfrak{K}=\left\{\left(T_{1} m, \ldots, T_{n} m\right) \mid m \in K\right\}$ is a convex subset of $X$, bounded away from zero. By the Hahn-Banach theorem there is $f \in X^{*}$ so that $|f(\mathfrak{K})| \geq 1$. Now, a linear functional in $X^{*}$ is of the form $\left(\mu_{1}, \ldots, \mu_{n}\right)$ where $\mu_{i} \in M\left(\rho^{-1}\right)(i=1, \ldots, n)$. Hence we have

$$
\left|\sum_{i=1}^{n}\left\langle T_{i} m, \mu_{i}\right\rangle\right| \geq 1 \quad(m \in K) .
$$

Let $\mathscr{U}$ be the filter of neighbourhoods of $e$ in $G$. By outer regularity of the measures in $M\left(\rho^{-1}\right)$ we may choose $U \in \mathscr{U}$ so that

$$
\max \left\{\int_{U \backslash\{e\}}\left|\frac{1}{\rho\left(r_{i} t r_{i}^{-1}\right)}+\frac{1}{\rho(t)}\right| d|\mu|(t) \mid i=1, \ldots, n\right\}<\frac{1}{n \rho(e)} .
$$

Then choose $V \in \mathscr{U}, V \subseteq U$ so that $r_{i}^{-1} V r_{i} \subseteq U \quad(i=1, \ldots, n)$. If $m \in K$ and $\operatorname{supp}(m) \subseteq V$, we have, using that $\left(T_{i} m\right)(e)=0 \quad(i=1, \ldots, n)$

$$
\begin{aligned}
\left|\left\langle T_{i} m, \mu_{i}\right\rangle\right| & =\left|\int_{G}\left[m\left(r_{i} t r_{i}^{-1}\right)-m(t)\right] d \mu_{i}(t)\right| \\
& \leq \int_{U \backslash\{e\}}\left|m\left(r_{i} t r_{i}^{-1}\right)-m(t)\right| d\left|\mu_{i}\right|(t) \\
& \leq\|m\| \int_{U \backslash\{e\}}\left|\frac{1}{\rho\left(r_{i} t r_{i}^{-1}\right)}+\frac{1}{\rho(t)}\right| d\left|\mu_{i}\right|(t) \\
& <\|m\| \frac{1}{n \rho(e)} \\
& \leq \frac{1}{n} .
\end{aligned}
$$

This contradicts (7).

\section{THE MAIN THEOREM}

In this section we establish the relationship between a BRAI in the diagonal ideal for $L^{1}(\omega)$ and certain invariant means for $G$. Since $L^{1}(\omega)$ has a BAI we have hereby characterized amenability. The description will be in terms of the auxillary weight defined in (6). As before $\nabla$ denotes the diagonal ideal for $L^{1}(\omega)$. 
Proposition 2.1. Suppose $\nabla$ has a BRAI. Then there is a left-invariant mean on $L^{\infty}\left(\Omega^{-1}\right)$.

Proof. By Proposition 1.7 it is enough to show that there is a left-invariant mean on $C_{\mathrm{uc}}\left(\Omega^{-1}\right)$. Let $\tilde{\omega}$ be as in $(5)$, let $m \in L^{\infty}(\tilde{\omega})$, and let $k \in \nabla$. We define the functional $\bar{k}(m)$ on $C_{\mathrm{uc}}\left(\Omega^{-1}\right)$ by

$$
\langle\varphi, \bar{k}(m)\rangle=\iint_{G \times G} m(t) \varphi(s) k\left(t s, s^{-1}\right) d s d t \quad\left(\varphi \in C_{\mathrm{uc}}\left(\Omega^{-1}\right)\right) .
$$

Since

$$
\begin{aligned}
& \iint_{G \times G} \frac{1}{\omega\left(t^{-1}\right)}|\varphi(s)| k\left(t s, s^{-1}\right) d s d t \\
& \quad=\iint_{G \times G} \frac{1}{\omega\left(t^{-1}\right)} \frac{\Omega(s)}{\omega(t s) \omega\left(s^{-1}\right)} \frac{|\varphi(s)|}{\Omega(s)} \omega(t s) \omega\left(s^{-1}\right)\left|k\left(t s, s^{-1}\right)\right| d s d t \\
& \quad \leq\|\varphi\|\|k\|,
\end{aligned}
$$

the functional $\bar{k}(m)$ is well defined and bounded, $\|\bar{k}(m)\| \leq\|m\|\|k\|$. Since $k \in \nabla$ we have $\int k\left(t s, s^{-1}\right) d s=0$ (a.e. $t$ ), so if $\varphi$ is constant it follows that

$$
\langle\varphi, \bar{k}(m)\rangle=0 \quad\left(m \in L^{\infty}(\tilde{\omega})\right) .
$$

Now if $r \in G$ we get

$$
\begin{aligned}
\left\langle\varphi \cdot \delta_{r}\right. & -\varphi, \bar{k}(m)\rangle \\
= & \iint_{G \times G} m(t)[\varphi(r s)-\varphi(s)] k\left(t s, s^{-1}\right) d s d t \\
= & \iint_{G \times G} m(t) \varphi(s)\left[k\left(t r^{-1} s, s^{-1} r\right)-k\left(t s, s^{-1}\right)\right] d s d t \\
= & \iint_{G \times G} \varphi(s)\left[m\left(r^{-1} t r\right) k\left(r^{-1} t s, s^{-1} r\right) \Delta(r)-m(t) k\left(t s, s^{-1}\right)\right] d s d t \\
= & \iint_{G \times G} \varphi(s)\left[m\left(r^{-1} t r\right)\left(\delta_{r, r^{-1}} * k\right)\left(t s, s^{-1}\right)-m(t) k\left(t s, s^{-1}\right)\right] d s d t \\
= & \left\langle\varphi,\left(Y^{r, r^{-1}} * k\right)(m)\right\rangle \\
& +\iint_{G \times G} \varphi(s)\left[m\left(r^{-1} t r\right)-m(t)\right]\left(\delta_{r, r^{-1}} * k\right)\left(t s, s^{-1}\right) d s d t .
\end{aligned}
$$

Denoting the last integral by $I(\varphi, m, r, k)$ we have

$$
\left.\left\langle\varphi \cdot \delta_{r}-\varphi, \bar{k}(m)\right\rangle=\left\langle\varphi, \overline{\left(Y^{r, r^{-1}} * k\right.}\right)(m)\right\rangle+I(\varphi, m, r, k) .
$$

Now suppose that $\varphi \in C_{\mathrm{uc}}\left(\Omega^{-1}\right)$. If $m$ is continuous with compact support it follows from Lemma 1.5 and Proposition 1.3 that the function $\Psi(s, t)=$ $m(s t) \varphi\left(t^{-1}\right)$ is in $L^{\infty}\left((\omega \times \omega)^{-1}\right) \cdot L^{1}(\omega \times \omega)$. If $\left(k_{y}\right)_{y \in \Gamma}$ is a BRAI for $\nabla$ we 
get

$$
\begin{aligned}
\left.\left\langle\varphi, \overline{\left(Y^{r, r^{-1}} * k_{\gamma}\right.}\right)(m)\right\rangle & =\iint m(t) \varphi(s)\left(Y^{r, r^{-1}} * k_{\gamma}\right)\left(t s, s^{-1}\right) d s d t \\
& =\iint m(s t) \varphi\left(t^{-1}\right)\left(Y^{r, r^{-1}} * k_{\gamma}\right)(s, t) d s, d t \\
& =\left\langle\Psi, Y^{r, r^{-1}} * k_{\gamma}\right\rangle .
\end{aligned}
$$

Since $\Psi \in L^{\infty}(\omega \times \omega) \cdot L^{1}(\omega \times \omega)$ and $\left(k_{\gamma}\right)_{\gamma \in \Gamma}$ is a BRAI for $\nabla$, this last term converges to $\left\langle\Psi, Y^{r, r^{-1}}\right\rangle$ along $\Gamma$, that is,

$$
\left.\left\langle\varphi, \overline{\left(Y^{r, r^{-1}} * k_{\gamma}\right.}\right)(m)\right\rangle \rightarrow m(e)(\varphi(r)-\varphi(e)) .
$$

Hence, if $m(e)=1$ we have, using (10) and (11),

$$
\begin{aligned}
& \underset{\gamma}{\lim \sup }\left|\left\langle\varphi \cdot \delta_{r}-\varphi, \bar{k}_{\gamma}(m)\right\rangle-(\varphi(r)-\varphi(e))\right| \\
& \quad \leq \limsup _{\gamma}|I(\varphi, m, r, k)| \\
& \quad \leq \underset{\gamma}{\lim \sup }\|\varphi\|\left\|m\left(r^{-1} \cdot r\right)-m\right\|\left\|\delta_{r, r^{-1}} * k_{\gamma}\right\| \\
& \quad \leq \Omega(r) d\|\varphi\|\left\|m\left(r^{-1} \cdot r\right)-m\right\|,
\end{aligned}
$$

where $d$ is the bound of $\left(k_{\gamma}\right)_{\gamma \in \Gamma}$.

Let $\mathscr{F} \subseteq G$ be a finite subset and let $\varepsilon>0$. Use Lemma 1.8 to choose $m_{\mathscr{F}, \varepsilon} \in C_{\mathrm{uc}}(\tilde{\omega})$ with $m_{\mathscr{F}, \varepsilon}(e)=1,\left\|m_{\mathscr{F}, \varepsilon}\right\| \leq 1$, and

$$
\left\|m\left(r^{-1} \cdot r\right)-m\right\|<\frac{\varepsilon}{\Omega(r) d} \quad(r \in \mathscr{F}) .
$$

Let $\Phi_{\mathscr{F}, \varepsilon}$ be the limit of some weak*-convergent subnet of $\left(\bar{k}_{\gamma}\left(m_{\mathscr{F}, \varepsilon}\right)\right)_{\gamma \in \Gamma}$. Then

$$
\left|\left\langle\varphi \cdot \delta_{r}-\varphi, \Phi_{\mathscr{F}, \varepsilon}\right\rangle-(\varphi(r)-\varphi(e))\right| \leq \varepsilon\|\varphi\| \quad(r \in \mathscr{F}) .
$$

If we order the pairs $(\mathscr{F}, \varepsilon)$ by the product order given by inclusion and the usual ordering of $\mathbf{R}_{+}$, we see that, if $\Phi$ is the limit of a weak*-convergent subnet of $\left(\Phi_{\mathscr{F}, \varepsilon}\right)$, then

$$
\left\langle\varphi \cdot \delta_{r}-\varphi, \Phi\right\rangle=\varphi(r)-\varphi(e) \quad(r \in G) .
$$

Hence, if we define the functional $M$ by

$$
\langle\varphi, M\rangle=\varphi(e)-\langle\varphi, \Phi\rangle \quad\left(\varphi \in C_{\mathrm{uc}}\left(\Omega^{-1}\right)\right),
$$

we see from (12) and (9) that $M$ is translation-invariant and satisfies $\left\langle\chi_{G}, M\right\rangle$ $=1$. As in [1, p. 240], we can obtain that $M$ is positive.

Proposition 2.2. If there is a left-invariant mean on $L^{\infty}\left(\Omega^{-1}\right)$, then there is a BRAI in $\nabla$.

Proof. Let $M$ be an invariant mean on $L^{\infty}\left(\Omega^{-1}\right)$. By Proposition 28.7 of [1] it suffices to prove that $\nabla^{* *}$ has a right unit for the Arens multiplication. 
Define $T: C_{\text {ruc }}\left((\omega \times \omega)^{-1}\right) \rightarrow C_{\text {ruc }}\left(\Omega^{-1}\right)$ by

$$
T \varphi(s)=\varphi(e, e)-\varphi\left(s, s^{-1}\right) \quad\left(\varphi \in C_{\mathrm{ruc}}\left((\omega \times \omega)^{-1}\right), s \in G\right) .
$$

We define a functional $N \in L^{\infty}\left((\omega \times \omega)^{-1}\right)^{*}$ by

$$
\langle f, N\rangle=\lim _{\gamma}\left\langle T\left(f \cdot e_{i,}\right), M\right\rangle,
$$

where $\left(e_{\gamma}\right)_{\gamma \in \Gamma}$ is some BAI for $L^{1}(\omega \times \omega)$ such that the net in $L^{\infty}\left((\omega \times \omega)^{-1}\right)^{*}$ given by $f \mapsto\left\langle T\left(f \cdot e_{y}\right), M\right\rangle$ has a weak*-limit. By Proposition 1.3 this is well defined. If we can show that $N$ is a right unit for $i^{* *}\left(\nabla^{* *}\right)$ we are done, since $i^{* *}$ is an algebra isomorphism.

Let $x \in L^{1}(\omega \times \omega)$, let $s, t \in G$, and let $u \in L^{\infty}\left((\omega \times \omega)^{-1}\right)$. Then, with $\varphi=u \cdot x$

$$
\left\langle u, x * Y^{s, t} \cdot N\right\rangle=\left\langle\varphi \cdot Y^{s, t}, N\right\rangle=\left\langle T\left(\varphi \cdot Y^{s, t}\right), M\right\rangle \text {. }
$$

Now

$$
T\left(\varphi, Y^{s, t}\right)(g)=\varphi(s, t)-\varphi(e, s t)-\left(\varphi\left(s g, g^{-1} t\right)-\varphi\left(g, g^{-1} s t\right)\right),
$$

for all $g \in G$, so, since $\varphi\left(s\left(s^{-1} g\right),\left(s^{-1} g\right)^{-1} t\right)=\varphi\left(g, g^{-1} s t\right)$, we get

$$
\begin{aligned}
\left\langle T\left(\varphi \cdot Y^{s, t}\right), M\right\rangle & =\langle g \mapsto \varphi(s, t)-\varphi(e, s t), M\rangle \\
& =\varphi(s, t)-\varphi(e, s t),
\end{aligned}
$$

where the first step follows from left-invariance of $M$, and the last from $M$ being a mean. Hence we have

$$
\left\langle u \cdot x * Y^{s, t}, N\right\rangle=\left\langle u, x * Y^{s, t}\right\rangle \text {. }
$$

Using Proposition 1.4, we then have $k \cdot N=\hat{k} \quad(k \in \nabla)$, where ${ }^{-}: L^{1}(\omega \times \omega) \rightarrow$ $L^{\infty}\left((\omega \times \omega)^{-1}\right)^{*}$ is the canonical embedding in the second dual. To finish, we show that $N \in i^{* *}\left(\nabla^{* *}\right)$. By exactness of

$$
0 \rightarrow \nabla^{* *} \stackrel{i^{* *}}{\rightarrow} L^{1}(\omega \times \omega)^{* *} \stackrel{\pi^{* *}}{\rightarrow} L^{1}(\omega)^{* *} \rightarrow 0,
$$

we must show that $\pi^{* *}(N)=0$. Since

$$
\pi^{*}\left(L^{\infty}\left(\omega^{-1}\right)\right) \cdot L^{1}(\omega \times \omega) \subseteq \pi^{*}\left(L^{1}(\omega) \cdot L^{\infty}\left(\omega^{-1}\right) \cdot L^{1}(\omega)\right),
$$

we have for all $f \in L^{\infty}\left(\omega^{-1}\right)$ and all $x \in L^{1}(\omega \times \omega)$ that

$$
T\left(\pi^{*}(f) \cdot x\right) \in T\left(\pi^{*}\left(C_{\mathrm{uc}}\left(\omega^{-1}\right)\right)\right)=\{0\} .
$$

Hence $\left\langle f, \pi^{* *}(N)\right\rangle=\lim _{\gamma^{\prime}}\left\langle T\left(\pi^{*}(f) \cdot e_{\gamma}\right), M\right\rangle=0$ for all $f \in L^{\infty}\left(\omega^{-1}\right)$.

We are now in the position to prove Theorem 0 .

Proof. The equivalence of (a) and (d) is the content of Propositions 2.1 and 2.2. The proofs of the remaining implications follow the proofs of the corresponding implications in Theorem 3.2 of [4], so we omit details. 
(c) $\Rightarrow(\mathrm{d})$ : The invariant mean is obtained as a weak*-cluster point of the net $\left(\mu_{i}\right)_{i \in I}$.

(d) $\Rightarrow$ (c): Let $M$ be the invariant mean, let $\mathscr{U}$ denote the filter of weak*neighbourhoods of $M$ in $L^{\infty}\left(\Omega^{-1}\right)$, and let $\mathscr{F}(G)$ be the set of finite subsets of $G$. Using the nondiscrete version of Lemma 3.1 of [4] we see that to each $V \in \mathscr{U}$, each $F \in \mathscr{F}(G)$, and each $n \in \mathbf{N}$ we can find $\mu \in V \cap L^{1}(\Omega)$ such that $\|\mu\| \leq\|M\|$ and $\max \left\{\| \delta_{r} \cdot \mu-\mu \mid r \in F\right\}<n^{-1}$. If we direct the $\mu$ 's so chosen by the product ordered set $\mathbf{N} \times \mathscr{F}(G) \times \mathscr{U}$, we get a bounded net in $L^{1}(\Omega)$ which satisfies the conditions of $(\mathrm{c})$.

(d) $\Rightarrow($ e): Since $\Omega$ is bounded away from zero, $G$ is clearly amenable. For $\alpha \in \mathbf{R}_{+}$define $E(\alpha)=\{g \mid \Omega(g)<\alpha\}$ and $F(\alpha)=G \backslash E(\alpha)$. Then $E(\alpha) F(\beta) \subseteq F(\beta / \alpha) \quad\left(\alpha, \beta \in \mathbf{R}_{+}\right)$. Using left-invariance one sees that if $F(\beta) \neq \varnothing$, then $0 \leq\left\langle\chi_{E(\alpha)}, M\right\rangle \leq \frac{\alpha}{\beta}\|M\|$. From the identity $\chi_{G}=\chi_{E(\alpha)}+\chi_{F(\alpha)}$ $\left(\alpha \in \mathbf{R}_{+}\right)$, the mean property $\left\langle\chi_{G}, M\right\rangle=1$, and $\lim _{\alpha \rightarrow+\infty}\left\|\chi_{F(\alpha)}\right\|=0$, it follows that $F(\alpha)=\varnothing$ for $\alpha$ sufficiently large.

(e) $\Rightarrow$ (d) : Straightforward, using that $c \leq \Omega \leq C$ for appropriate constants $c, C \in] 0 \infty[$.

(b) $\Leftrightarrow(\mathrm{e})$ : This follows from the equivalence of (a) and (e), with $\Omega$ replacing $\omega$.

Acknowledgment. Most of the research contained in this paper was done during the author's attendance at the 'Semester on Automatic Continuity and Banach Algebras' held at the University of Leeds, Spring 1987. The author wishes to thank the Danish Natural Science Research Council and the United Kingdom Science and Engineering Research Council for supporting his participation.

\section{REFERENCES}

1. F. F. Bonsall and J. Duncan, Complete normed algebras, Springer-Verlag, New York 1973.

2. P. C. Curtis, Jr. and R. J. Loy, The structure of amenable Banach algebras, J. London Math. Soc. (to appear).

3. F. P. Greenleaf, Invariant means on topological groups, Van Nostrand, New York, 1969.

4. N. Grønbæk, Amenability of weighted discrete convolution algebras on cancellative semigroups, Proc. Royal Soc. Edinburgh 110A (1988), 351-360.

5. B. E. Johnson, Cohomology in Banach algebras, Mem. Amer. Math. Soc. No. 127 (1972).

6. A. Ya. Khelemskii, Flat Banach modules and amenable algebras, Trans. Moscow Math. Soc. (AMS Transl. 1985) 47 (1984), 199-244.

KobenhaVNS UNiversitets Matematiske Institut, Universitetsparken 5, 2100 KobenHAVN 0, DenMaRK 